(2) Open Access Full Text Article

\title{
In vitro and in vivo efficacy of afatinib as a single agent or in combination with gemcitabine for the treatment of nasopharyngeal carcinoma
}

This article was published in the following Dove Press journal:

Drug Design, Development and Therapy

31 March 2016

Number of times this article has been viewed

\author{
Cong Xue' \\ Ying Tian ${ }^{2}$ \\ Jing Zhang ${ }^{3}$ \\ Yuanyuan Zhao' \\ Jianhua Zhan ${ }^{2}$ \\ Wenfeng Fang' \\ Li Zhang'
}

'Department of Medical Oncology, ${ }^{2}$ Department of Research, Sun Yat-Sen University Cancer Center, State Key Laboratory of Oncology in South China, Collaborative Innovation Center of Cancer Medicine, ${ }^{3}$ Department of Medical Oncology, The First Affiliated Hospital of Guangzhou Traditional Chinese Medicine University, Guangzhou, Guangdong, People's Republic of China
Correspondence: Li Zhang Department of Medical Oncology, Sun Yat-Sen University Cancer Center, State Key Laboratory of Oncology in South China, Collaborative Innovation Center of Cancer Medicine, 65I Dongfeng East Road, Guangzhou, 510060, People's Republic of China

Tel +862087343689

Fax +86 2087343565

Email zhangli@sysucc.org.cn
Purpose: Epidermal growth factor receptor (EGFR) is usually overexpressed in nasopharyngeal carcinoma (NPC). We tested the antitumor effects of irreversible ErbB family inhibitor afatinib on human NPC using in vitro and in vivo models.

Materials and methods: The effect of afatinib on NPC cells was evaluated using the Cell Counting Kit 8 (CCK8) assay, flow cytometry, and Western blot analyses. The effect of afatinib, as either a single agent or in combination with gemcitabine (GEM), on tumor growth was determined using NPC tumor xenografts in mice.

Results: Afatinib inhibited cell growth in all three NPC cell lines tested in a dose-dependent manner. Afatinib promoted cell cycle arrest at the S and G2/M phases, and it significantly inhibited epidermal growth factor (EGF)-induced activation of EGFR and its downstream signaling factors. Co-treatment with afatinib and GEM more effectively inhibited tumor growth than either drug alone but was associated with increased toxicity.

Conclusion: Afatinib induced cell cycle arrest and inhibited the proliferation of NPC cell lines. Afatinib in combination with GEM demonstrated significant antitumor effect in an NPC xenograft model. The administration of afatinib with GEM in NPC needs to be modified in order to be effective and tolerable.

Keywords: nasopharyngeal carcinoma, EGFR, afatinib, gemcitabine, preclinical

\section{Introduction}

Nasopharyngeal carcinoma (NPC) has a distinct geographic distribution, with low morbidity in most areas worldwide (generally $<1 / 100,000) .{ }^{1}$ Eighty percent of all NPC cases occur in the People's Republic of China, with the highest incidence rates in South China. ${ }^{2}$ NPC-related morbidity in the northern region of the People's Republic of China is comparable to that observed in the remaining parts of the world. The incidence of NPC in South China is the highest in the world $(\sim 20-30 / 100,000$ for nearly 30 years). ${ }^{3}$

NPC is very sensitive to radiotherapy, and radiotherapy is the treatment of choice for early nonmetastatic NPC. ${ }^{4}$ With advances in imaging and radiation therapy, the 5-year local control rate of nonmetastatic NPC has risen to $94.9 \%$, and the 5-year relapse-free survival rate has reached $76.7 \%$. The main cause of treatment failure of NPC is distant metastases, whereby $\sim 20 \%$ of patients with locally advanced disease present with remote metastasis. ${ }^{5}$

More than $90 \%$ of NPCs are undifferentiated carcinomas, which show relatively good reactivity to chemotherapy. Many cytotoxic chemotherapeutic agents have been tested for NPC, and currently, platinum-based treatment is the basic regimen for 
palliative care. For salvage therapy after failure of platinum regimen, yew, gemcitabine (GEM), and others are effective. ${ }^{6,7}$ Despite the relatively high efficacy of these therapies and the eventual availability of various new drugs, the survival of patients with late NPC has not significantly improved, with progression-free survival (PFS) at about 5.6-10.6 months and overall survival (OS) at about 7.6-19.6 months. ${ }^{6,8-10}$ Identification of novel drugs, especially targeted therapies, is needed to improve the mortality rate in patients with advanced NPC.

Epidermal growth factor receptor (EGFR) is overexpressed in $73 \%-89 \%$ of NPC patients, contributing to an increased risk of metastasis and decreased OS. ${ }^{11-14}$ However, EGFR mutations are rarely detected in NPC, ${ }^{15}$ suggesting that EGFR mutations might not be a critical factor in NP carcinogenesis. The first-generation EGFR-tyrosine kinase inhibitors (TKIs) were effective in patients with EGFR mutations, which may explain why previous studies found that EGFR-TKIs only stabilized the disease in most patients with refractory metastatic NPC. ${ }^{16,17}$

Compared to the first-generation reversible EGFR-TKIs gefitinib and erlotinib, afatinib is a potent irreversible ErbB inhibitor that blocks the activity of all kinases related to ErbB family members, including wild-type and mutated EGFR (eg, T790M) and human epidermal growth factor receptor 2 (HER2) ${ }^{18}$ Owing to its efficacy in the EGFR mutation-associated non-small-cell lung cancer (NSCLC), afatinib was approved as a first-line therapy for advanced NSCLC with mutant EGFR. ${ }^{19}$

Recent studies have focused on whether afatinib can be applied to EGFR wild-type tumors, and some preclinical and clinical data have shown that afatinib does suppress the growth of cancer cells with wild-type EGFR. ${ }^{19,20}$ There are ongoing clinical studies investigating the antitumor efficacy of afatinib in multiple solid tumors with ErbB deregulations. ${ }^{21-23}$ Similar to NPC, overexpression of EGFR is commonly found in squamous cell cancer of the head and neck (SCCHN). Young et $\mathrm{al}^{24}$ demonstrated that the efficacy of afatinib was comparable to that of cetuximab in SCCHN cell lines and xenograft models. A Phase II study ${ }^{25}$ comparing the efficacy of cetuximab with afatinib in refractory recurrent SCCHN patients showed similar response and survival rates. Taken together, these data provided the rationale for investigating the use of afatinib for NPC. A Phase III clinical trial compared afatinib with methotrexate (MTX) as a second-line treatment for patients with refractory metastatic head and neck cancer after failure of platinum treatment and found that afatinib significantly prolonged PFS relative to MTX (2.6 months vs 1.7 months; $P=0.03$ ). In addition, afatinib improved the objective response rate (ORR: $10.2 \%$ vs $5.6 \% ; P=0.10)$ and the disease control rate $(49.1 \%$ vs
$38.5 \% ; P=0.04$ ), with tolerable side effects, ${ }^{26}$ relative to MTX. These results showed that afatinib as a second-line therapeutic regimen had a better curative effect than MTX in refractory head and neck cancer and that its application in head and neck cancer required further exploration.

In this study, we explored whether afatinib has curative effects in preclinical studies of NPC, which will shed light on its use for other applications. We selected GEM for combination with afatinib because GEM is a novel and effective agent for the treatment of NPC and is currently being investigated as a first-line therapy in metastatic NPC. . $^{6-10,27}$ Moreover, former in vitro studies showed that combination treatment with afatinib and GEM had synergistic effects. ${ }^{23}$ We hypothesized that combination therapy with afatinib and GEM may have enhanced the antitumor activity relative to the individual administration of these two agents.

\section{Materials and methods Cell lines}

All cell line experiments were approved by the Ethical Committee of Sun Yat-sen University Cancer Center. Three poorly differentiated human NPC cell lines were studied: HNE-1, CNE-2, and SUNE-1. HNE-1 was kindly provided by Professor Kaitai Yao (Southern Medical University, Guangzhou, People's Republic of China). CNE-2 and SUNE-1 were kindly provided by Professor Musheng Zeng (Sun Yat-Sen University Cancer Center, Guangzhou, People's Republic of China). Cells were cultured in RPMI 1640 supplemented with $10 \%$ fetal bovine serum (FBS), 100 units $/ \mathrm{mL}$ penicillin, and 100 units $/ \mathrm{mL}$ streptomycin at $37^{\circ} \mathrm{C}$ in a humidified atmosphere containing $5 \% \mathrm{CO}_{2}$. Logarithmically growing cells were used in the experiments.

\section{Reagents and drug preparation}

Afatinib was kindly provided by Boehringer Ingelheim Pharmaceuticals (Ingelheim am Rhein, Germany). GEM (Gemzar; Eli-lilly Pharmaceuticals, Indiana, IN, USA) was obtained as a commercial product from our hospital pharmacy.

Afatinib was dissolved in dimethylsulfoxide at a stock concentration of $10 \mathrm{mM}$ and stored at $-20^{\circ} \mathrm{C}$. For in vivo studies, afatinib was dissolved in plain water at a concentration of $1.25 \mathrm{mg} / \mathrm{mL}$. GEM was dissolved in plain water at a stock concentration of $100 \mathrm{mM}$ and stored at $-20^{\circ} \mathrm{C}$.

\section{Proliferation assay}

Tumor cells were cultured in 96-well plates at an appropriate density per well. Varying concentrations of afatinib, GEM, and the combination were added to the cells 24 hours after plating and incubated for 72 hours, followed by Cell Counting Kit 8 
(CCK8; Dojindo, Tokyo, Japan) assay. Afatinib and GEM were added at the same concentration when in the combination assay (eg, both $0.625 \mu \mathrm{M}$ ). The optical density was measured at $450 \mathrm{~nm}$ on an enzyme-linked immunosorbent assay reader (SpectraMax M5; Molecular Devices, Sunnyvale, CA, USA). The half-maximal inhibitory concentration $\left(\mathrm{IC}_{50}\right)$ value was defined as the concentration resulting in $50 \%$ of cell growth inhibition relative to untreated control cells. The assay was performed in triplicate in more than three independent experiments. The combination index (CI) was calculated by CalcuSyn (Biosoft, Cambridge, UK). CI $<1$ indicates synergism; $\mathrm{CI}=1$ indicates additive effects; $\mathrm{CI}>1$ indicates antagonism. ${ }^{28}$

\section{Cell cycle analysis}

Cells were seeded into six-well plates containing RPMI $1640 / 10 \%$ FBS with or without various concentrations of afatinib. Following 48-hour incubation, cells were harvested and fixed with $70 \%$ ethanol and stored at $-20^{\circ} \mathrm{C}$. Tumor cells were incubated with propidium iodide/RNase buffer (Becton Dickinson, Bedford, MA, USA) for 30 minutes at room temperature in the dark. Analysis was performed using the FACScan flow cytometer (Beckman Coulter Inc., Brea, CA, USA) and CellQuest Pro software (Becton, Dickinson and Company, Franklin Lakes, NJ, USA).

\section{Western blotting}

Cells were treated with afatinib for 24 hours and then stimulated with $10 \mathrm{nM}$ epidermal growth factor (EGF) (Cell Signaling Technology, Beverley, MA, USA) for 30 minutes before harvest. Cells were harvested and lysed in cell lysis buffer (Cell Signaling Technology). Proteins were resolved on sodium dodecyl sulfate polyacrylamide gel electrophoresis and transferred onto polyvinylidene fluoride membrane (Hoffman-La Roche Ltd., Basel, Switzerland). The membranes were incubated overnight at $4^{\circ} \mathrm{C}$ with primary antibodies. After being incubated with the appropriate horseradish peroxidase-conjugated secondary antibody for 1 hour at room temperature, bands were detected using an enhanced chemiluminescence system (Cell Signaling Technology). The expression of phosphorylated-EGFR (pEGFR), pAKT, and p-extracellular regulated kinase ( $\mathrm{pERK}$ ) was analyzed to detect the activation of EGFR and its downstream pathways (all antibodies were purchased from Cell Signaling Technology). The quantitative analysis was performed using ImageJ (National Institutes of Health, Bethesda, MD, USA).

\section{Tumor xenograft studies}

Six- to 8-week-old male BALB/c nude mice weighing $\sim 16-18 \mathrm{~g}$ were maintained under specific pathogen-free conditions. All animal experiments were conducted in accordance with the United Kingdom Co-ordinating Committee on Cancer Research Guidelines for the welfare of animals in experimental neoplasia. All animal experiments were approved by the Experimental Animal Ethical Committee of Sun Yat-sen University. HNE- 1 cells $\left(1 \times 10^{7}\right.$ cells resuspended in $0.2 \mathrm{~mL}$ of $0.9 \% \mathrm{NaCl}$ solution) were inoculated subcutaneously into the right flank of nude mice. When tumors reached $\sim 150 \mathrm{~mm}^{3}$, animals were randomly assigned to one of four groups ( $n=8$ per group): afatinib group, GEM group, combination group, and control group. Afatinib (12.5 mg/kg) was administered by oral gavage for 5 days a week. Intraperitoneal injection of GEM $(100 \mathrm{mg} / \mathrm{kg}$ ) was dosed with $0.9 \% \mathrm{NaCl}$ every week. $\mathrm{NaCl}$ $(0.9 \%, 10 \mathrm{~mL} / \mathrm{kg})$ was administered by oral gavage for 5 days a week, and intraperitoneal injection of $0.9 \% \mathrm{NaCl}$ was administered every week as control treatment. Mice body weight and tumor size were measured and recorded every 3 days. Tumor volume was calculated using the following equation: volume $\left(\mathrm{mm}^{3}\right)=$ length $\times$ width $^{2} \times 0.5$. After 4 weeks of treatment, mice were sacrificed, and tumors were harvested, fixed in $10 \%$ buffered formalin, and embedded in paraffin.

\section{Statistics}

Statistical analyses were performed using SPSS Version 16.0 software (SSPS Inc., Chicago, IL, USA). Data were expressed as mean \pm standard deviation. Comparisons in the in vivo studies were analyzed by the unpaired $t$-test with Welch correction. Two-sided $P<0.05$ was considered statistically significant.

\section{Results}

\section{Afatinib inhibited proliferation in NPC cell lines and showed synergistic effect with GEM}

Three human NPC cell lines - CNE-2, HNE-1, and SUNE-1 - were treated with afatinib $(0-10 \mu \mathrm{M})$ for 72 hours. Afatinib demonstrated a steep dose-response relationship, with dose-dependent growth inhibition in all cell lines (Figure 1). The $\mathrm{IC}_{50}$ values for afatinib in HNE-1, CNE-2, and SUNE-1 cell lines were $4.41 \pm 0.73 \mu \mathrm{M}, 2.81 \pm 0.35 \mu \mathrm{M}$, and $6.93 \pm 0.54 \mu \mathrm{M}$, respectively. When combined with GEM, the CI values were separately $0.293,0.435$, and 0.654 when the concentrations were $0.625 \mu \mathrm{M}, 1.25 \mu \mathrm{M}$, and $2.5 \mu \mathrm{M}$ for both drugs, which indicates that synergistic effect exists when afatinib is combined with GEM.

\section{Afatinib arrested the cell cycle at $S$ and G2/M phases}

The effects of afatinib on the cell cycle of NPC cells were determined by flow cytometry. HNE-1 cells were treated 


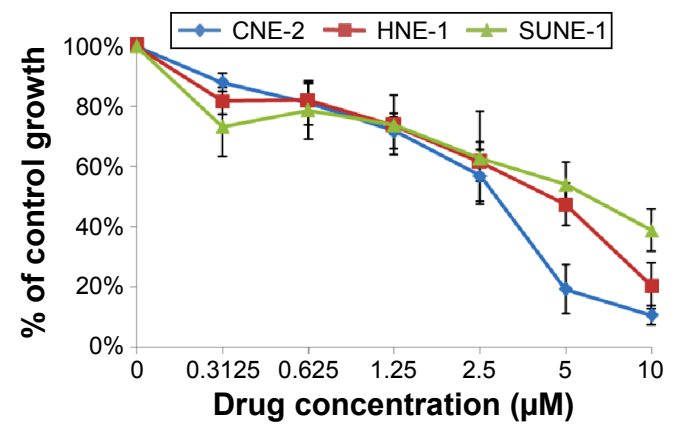

Figure I Inhibitory effect of afatinib on growth of NPC cell lines. Notes: Cancer cell proliferation was calculated as a percentage of growth of the control cells. Each point represents the mean of triplicate values \pm standard deviation.

Abbreviations: CNE,HNE,SUNE: three human NPC cell lines; NPC, nasopharyngeal carcinoma.

with various concentrations $(0 \mu \mathrm{M}, 0.625 \mu \mathrm{M}, 1.25 \mu \mathrm{M}$, $2.5 \mu \mathrm{M}$, and $5 \mu \mathrm{M}$ ) of afatinib for 48 hours, resulting in a dose-dependent increase in the percentage of cells in the $S$ and $\mathrm{G} 2 / \mathrm{M}$ phases and a concomitant decrease in the proportion of cells in the G0/G1 phase (Figure 2).

\section{Afatinib inhibited the EGFR signaling pathway}

We examined the activity of EGFR and its downstream targets AKT and ERK in HNE-1 cells treated with afatinib. As shown in Figure 3, after exposure to afatinib for 24 hours,
EGF-induced phosphorylation of EGFR, AKT, and ERK was suppressed by afatinib. However, afatinib treatment did not affect the levels of p-EGFR, p-AKT, and p-ERK in the absence of EGF stimulation. The quantitative analysis is provided in Figure $\mathrm{S} 1$.

\section{Antitumor activity of afatinib in vivo}

We evaluated the efficacy of afatinib in HNE-1 xenograft mouse model, both as a single agent and in combination with GEM. As shown in Figure 4A, afatinib modestly inhibited NPC tumor growth in the HNE-1 xenograft model. In the human NPC cell line xenograft model, combination treatment with afatinib and GEM significantly inhibited tumor growth more than that obtained by the two treatments alone. However, toxicity was more severe, as evidenced by a greater decrease in body weight, in the combination group than in the other treatment groups (Figure 4B).

\section{Discussion}

This preclinical study is the first to investigate the efficacy of afatinib in NPC, and our findings suggest that afatinib is a promising agent for the treatment of NPC. The $\mathrm{IC}_{50}$ values of afatinib were found to be around 3-7 $\mu \mathrm{M}$ in three poorly differentiated human NPC cell lines, suggesting that these cell lines are marginally sensitive to afatinib monotherapy.
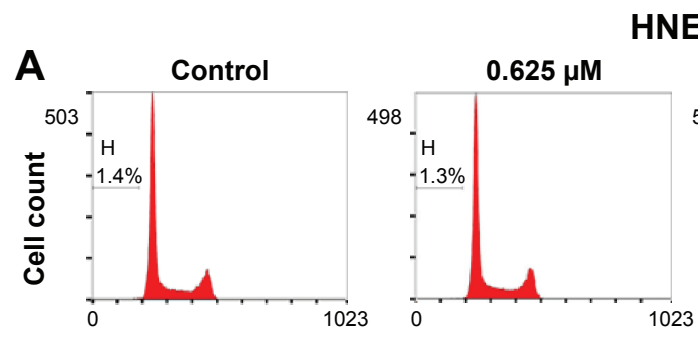

HNE-1 cell cycle for 48 hours
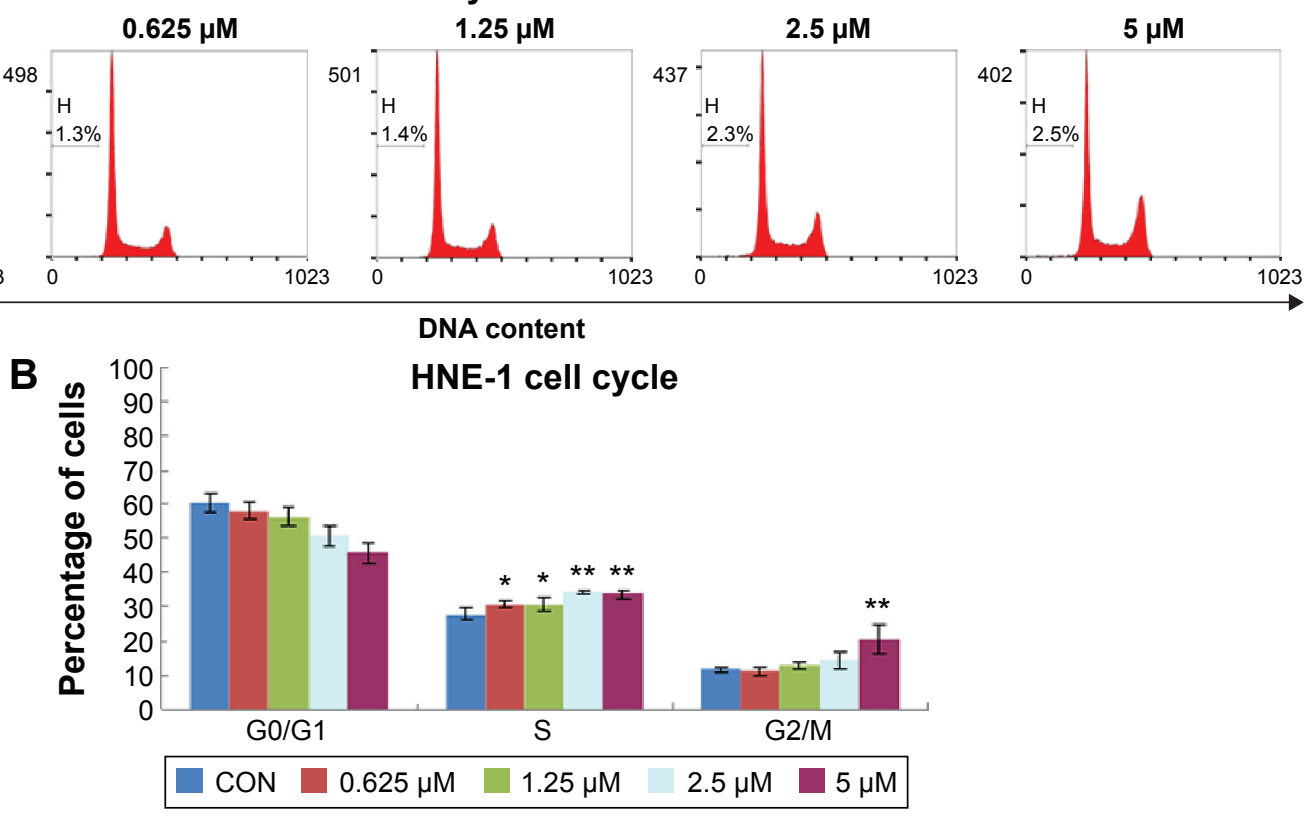

Figure 2 Afatinib induced cell cycle arrest at GI phase in HNE-I cell line.

Notes: (A) Cell cycles were assessed by flow cytometry and one representative experiment is shown. (B) Statistical results are shown in the graph. Values are presented as mean of triplicate \pm SD. *Represents $P<0.05$, **represents $P<0.01$.

Abbreviations: CON, control; H, percentage of sub-GI phase; HNE-I, human NPC cell line; NPC, nasopharyngeal carcinoma; SD, standard deviation. 


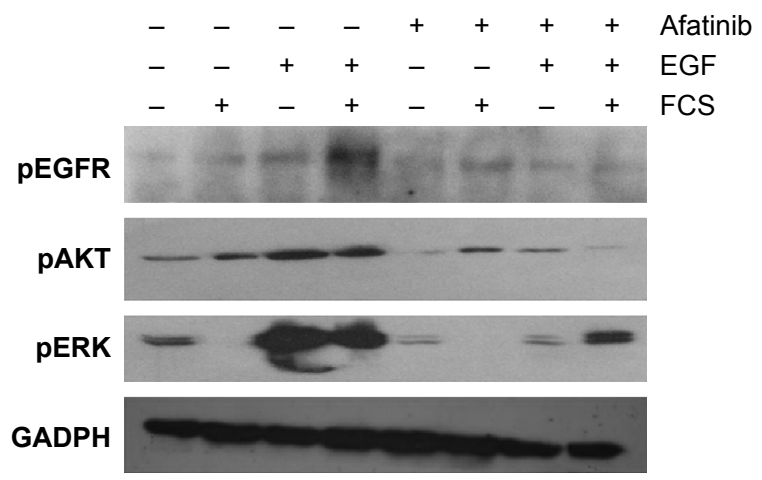

Figure 3 Effect of afatinib on levels of phosphorylated forms of EGFR, AKT, and ERK in the HNE-I cell line.

Notes: Some cells were incubated with $10 \mathrm{ng} / \mathrm{mL}$ EGF for 30 minutes. Afatinib $(5 \mu \mathrm{M})$ was administered as described in the "Materials and methods" section. Abbreviations: EGF, epidermal growth factor; EGFR, epidermal growth factor receptor; ERK, extracellular regulated protein kinases; FCS, fetal calf serum; GADPH, glyceraldehyde-3-phosphate dehydrogenase; HNE-I, human NPC cell line; NPC, nasopharyngeal carcinoma; p, phosphorylated.

In the Phase I trials of afatinib monotherapy for advanced solid tumors, different doses of afatinib lower than the maximum tolerated dose achieved an average steady-state maximum concentration $\left(C_{\text {max, ss }}\right)$ of $63.4 \mathrm{ng} / \mathrm{mL}(\sim 0.13 \mu \mathrm{M})$. We observed that the growth inhibition rate of this dose of afatinib was only about $10 \%-20 \%$. The $\mathrm{IC}_{50}$ of all three cell lines after conversion was $1.37-3.38 \mu \mathrm{g} / \mathrm{mL}$ (the molecular weight of afatinib is $\sim 486 \mathrm{~g} / \mathrm{mol}$ ), and this drug dosage is much higher than the human tolerated blood drug concentration of afatinib. Western blot data also showed that afatinib did not inhibit the EGFR signaling pathway when EGFR was not overactive. In tumors not driven by EGFR mutations, such as gastric cancer, pancreatic cancer, and SCCHN, the combination of afatinib and other agents or radiotherapy might have better response than afatinib alone. ${ }^{21-23}$ Therefore, we believe that the currently used dose of afatinib may fail to produce satisfactory curative effects as monotherapy in patients with advanced NPC, whereas its use in combination with other drugs (targeted drugs or chemotherapy drug) may be ideal.

Previously, preclinical studies ${ }^{22,23}$ reported that afatinib mainly inhibited the growth of tumor cell lines by causing retardation of the cell's $\mathrm{G}_{1}$ phase. Because chemotherapy drugs mainly act on cells in the proliferating and division phase, there is no appropriate theoretical basis to support the existence of synergic efficiency during concurrent application of small molecule inhibitors and chemotherapy drugs. In addition, both in vitro and in vivo experiments have shown no positive results. ${ }^{29}$ In our study, cell cycle analysis demonstrated that afatinib induced cell arrest in the G2/M and S phases. This is a very interesting finding, as it provides some support for the use of combination treatments with afatinib and chemotherapeutic agents for NPC patients. Because GEM is an S phase-specific cytotoxic agent, we predicted that this combination might increase efficacy. Our in vivo experiments using combination treatment with afatinib and GEM confirmed this hypothesis.
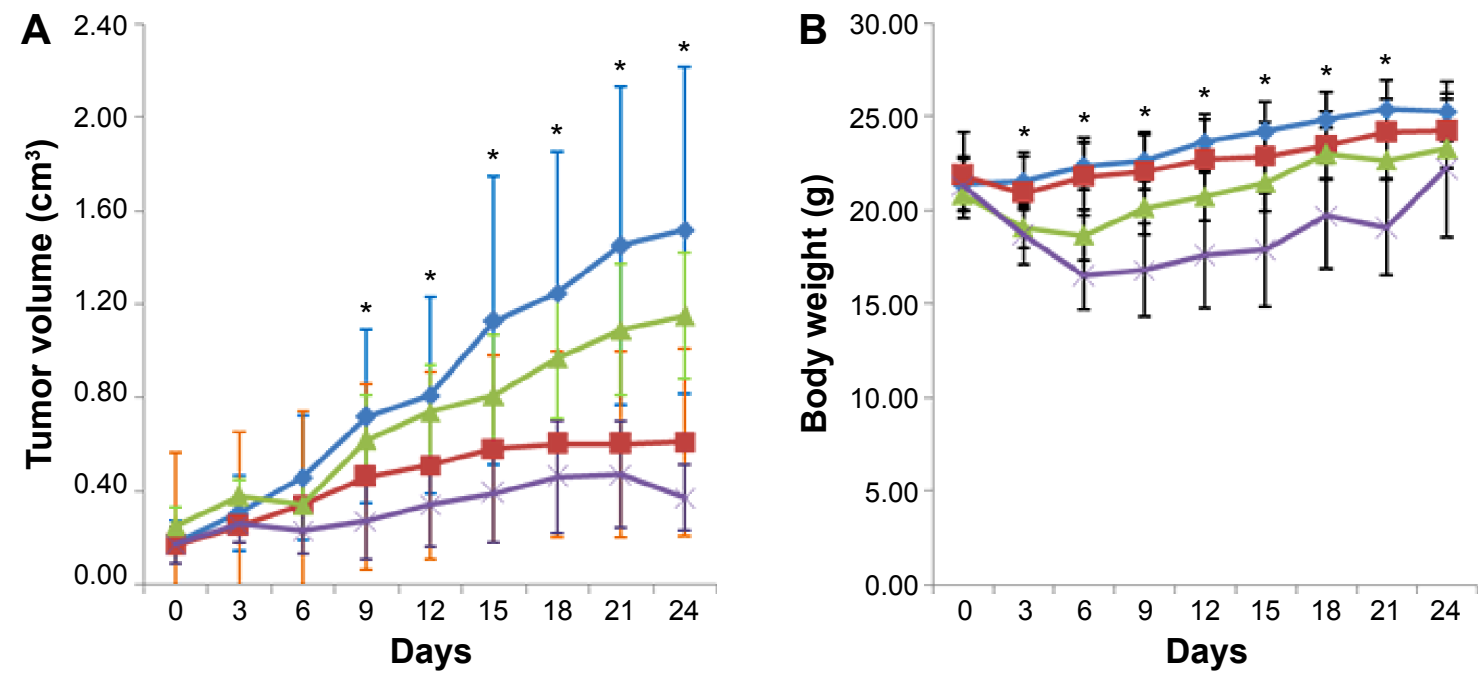

$\multimap$ Control $\because-$ Afatinib $\longleftarrow$ Gemcitabine $\longleftarrow$ Combination

Figure 4 Effect of afatinib, gemcitabine, or combination on the growth of HNE-I xenografts.

Notes: (A) Tumor volume of HNE-I xenografts. Mean tumor volume is shown. (B) Body weight of HNE-I xenografts. Mean body weight is shown. Data points represent the mean value from six to nine mice; Bars represent the standard deviation. $* P<0.05$ versus control.

Abbreviations: HNE-I, human NPC cell line; NPC, nasopharyngeal carcinoma. 
The human NPC xenograft model demonstrated good sensitivity toward afatinib monotherapy treatment at $12.5 \mathrm{mg} / \mathrm{kg}$. Afatinib in combination with GEM resulted in significant inhibition of tumor growth. However, more weight loss was observed in the combination group, showing that toxicity might limit the clinical applicability of concurrent therapy. To achieve safe and effective results in subsequent clinical studies, the dosages of both afatinib and GEM need to be further explored.

Another option for including afatinib in treatment regimens for NPC is sequential therapy. Previous studies have shown that most EGFR-TKIs induce G0/G1 cell cycle arrest, which might protect cells from the cytotoxic effects of cell cycle phase-dependent chemotherapy agents. ${ }^{29}$ Sequential administration of EGFR-TKIs following chemotherapy may provide greater efficacy than concurrent administration in NSCLC. ${ }^{30,31}$ In other preclinical models, ${ }^{32}$ sequential administrations of two potent agents could delay disease progression. In our preclinical study, afatinib and GEM, both active drugs against NPC, were administered concomitantly. The combination treatment, although effective, induced more toxicity than either agent alone did. On the basis of these observations, we suggest modifying the method of administration. Further studies are needed to evaluate the full potential of this combination.

In conclusion, afatinib effectively arrested the growth of NPC cell lines in vitro. Data from the NPC xenograft model suggested that concurrent administration of afatinib with GEM might lead to significant efficacy but at the cost of enhanced toxicity. Combination therapy of GEM along with afatinib might be an effective treatment option for NPC once alternative dosage or methods of administration (schedules) are optimized in model systems, resulting in a larger therapeutic window for this combination.

\section{Acknowledgments}

Cong Xue and Ying Tian are joint first authors. This study was supported by a Chinese National Natural Science Foundation project (grant numbers 81372502 and 81201917), the Specialized Research Fund for the Doctoral Program of Higher Education (20120171120116), the Young Teacher Training Program of Sun Yat-Sen University (14ykpy38), and the Outstanding Young Talent Cultivation Project of Sun Yat-Sen University Cancer Center (04140701).

\section{Disclosure}

The authors report no conflicts of interest in this work.

\section{References}

1. Cancer incidence in five continents. Volume VII. IARC Sci Publ. 1997:i-xxxiv, 1-1240.

2. Wei KR, Zheng RS, Zhang SW, Liang ZH, Ou ZX, Chen WQ. Nasopharyngeal carcinoma incidence and mortality in China in 2010. Chin J Cancer. 2014;33(8):381-387.

3. Zhang LF, Li YH, Xie SH, et al. Incidence trend of nasopharyngeal carcinoma from 1987 to 2011 in Sihui County, Guangdong Province, South China: an age-period-cohort analysis. Chin J Cancer. 2015;34(8):350-357.

4. Ma BB, Hui EP, Chan AT. Systemic approach to improving treatment outcome in nasopharyngeal carcinoma: current and future directions. Cancer Sci. 2008;99(7):1311-1318.

5. Xiao WW, Huang SM, Han F, et al. Local control, survival, and late toxicities of locally advanced nasopharyngeal carcinoma treated by simultaneous modulated accelerated radiotherapy combined with cisplatin concurrent chemotherapy: long-term results of a phase 2 study. Cancer. 2011;117(9):1874-1883.

6. Ngan RK, Yiu HH, Lau WH, et al. Combination gemcitabine and cisplatin chemotherapy for metastatic or recurrent nasopharyngeal carcinoma: report of a phase II study. Ann Oncol. 2002;13(8):1252-1258.

7. Tan EH, Khoo KS, Wee J, et al. Phase II trial of a paclitaxel and carboplatin combination in Asian patients with metastatic nasopharyngeal carcinoma. Ann Oncol. 1999;10(2):235-237.

8. Zhang L, Zhang Y, Huang PY, Xu F, Peng PJ, Guan ZZ. Phase II clinical study of gemcitabine in the treatment of patients with advanced nasopharyngeal carcinoma after the failure of platinum-based chemotherapy. Cancer Chemother Pharmacol. 2008;61(1):33-38.

9. Ma BB, Hui EP, Wong SC, et al. Multicenter phase II study of gemcitabine and oxaliplatin in advanced nasopharyngeal carcinoma correlation with excision repair cross-complementing-1 polymorphisms. Ann Oncol. 2009;20(11):1854-1859.

10. Wang CC, Chang JY, Liu TW, Lin CY, Yu YC, Hong RL. Phase II study of gemcitabine plus vinorelbine in the treatment of cisplatin-resistant nasopharyngeal carcinoma. Head Neck. 2006;28(1):74-80.

11. Fujii M, Yamashita T, Ishiguro R, Tashiro M, Kameyama K. Significance of epidermal growth factor receptor and tumor associated tissue eosinophilia in the prognosis of patients with nasopharyngeal carcinoma. Auris Nasus Larynx. 2002;29(2):175-181.

12. Ma BB, Poon TC, To KF, et al. Prognostic significance of tumor angiogenesis, Ki 67, p53 oncoprotein, epidermal growth factor receptor and HER2 receptor protein expression in undifferentiated nasopharyngeal carcinoma - a prospective study. Head Neck. 2003;25(10): 864-872.

13. Sheen TS, Huang YT, Chang YL, et al. Epstein-Barr virus-encoded latent membrane protein 1 co-expresses with epidermal growth factor receptor in nasopharyngeal carcinoma. Jpn J Cancer Res. 1999; 90(12):1285-1292.

14. Chua DT, Nicholls JM, Sham JS, Au GK. Prognostic value of epidermal growth factor receptor expression in patients with advanced stage nasopharyngeal carcinoma treated with induction chemotherapy and radiotherapy. Int J Radiat Oncol Biol Phys. 2004;59(1):11-20.

15. Lee SC, Lim SG, Soo R, et al. Lack of somatic mutations in EGFR tyrosine kinase domain in hepatocellular and nasopharyngeal carcinoma. Pharmacogenet Genomics. 2006;16(1):73-74.

16. Chua DT, Wei WI, Wong MP, Sham JS, Nicholls J, Au GK. Phase II study of gefitinib for the treatment of recurrent and metastatic nasopharyngeal carcinoma. Head Neck. 2008;30(7):863-867.

17. Ma B, Hui EP, King A, et al. A phase II study of patients with metastatic or locoregionally recurrent nasopharyngeal carcinoma and evaluation of plasma Epstein-Barr virus DNA as a biomarker of efficacy. Cancer Chemother Pharmacol. 2008;62(1):59-64.

18. Li D, Ambrogio L, Shimamura T, et al. BIBW2992, an irreversible EGFR/HER2 inhibitor highly effective in preclinical lung cancer models. Oncogene. 2008;27(34):4702-4711. 
19. Sequist LV, Yang JC, Yamamoto N, et al. Phase III study of afatinib or cisplatin plus pemetrexed in patients with metastatic lung adenocarcinoma with EGFR mutations. J Clin Oncol. 2013;31(27):3327-3334.

20. Miller VA, Hirsh V, Cadranel J, et al. Afatinib versus placebo for patients with advanced, metastatic non-small-cell lung cancer after failure of erlotinib, gefitinib, or both, and one or two lines of chemotherapy (LUX-Lung 1): a phase 2b/3 randomised trial. Lancet Oncol. 2012;13(5):528-538.

21. Schutze C, Dorfler A, Eicheler W, et al. Combination of EGFR/HER2 tyrosine kinase inhibition by BIBW 2992 and BIBW 2669 with irradiation in FaDu human squamous cell carcinoma. Strahlenther Onkol. 2007;183(5):256-264.

22. Khelwatty SA, Essapen S, Seddon AM, Modjtahedi H. Growth response of human colorectal tumour cell lines to treatment with afatinib (BIBW2992), an irreversible erbB family blocker, and its association with expression of HER family members. Int J Oncol. 2011; 39(2):483-491.

23. Ioannou N, Dalgleish AG, Seddon AM, et al. Anti-tumour activity of afatinib, an irreversible ErbB family blocker, in human pancreatic tumour cells. Br J Cancer. 2011;105(10):1554-1562.

24. Young NR, Soneru C, Liu J, et al. Afatinib efficacy against squamous cell carcinoma of the head and neck cell lines in vitro and in vivo. Target Oncol. 2015;10(4):501-508.

25. Seiwert TY, Fayette J, Cupissol D, et al. A randomized, phase II study of afatinib versus cetuximab in metastatic or recurrent squamous cell carcinoma of the head and neck. Ann Oncol. 2014;25(9):1813-1820.
26. Machiels JP, Haddad RI, Fayette J, et al. Afatinib versus methotrexate as second-line treatment in patients with recurrent or metastatic squamous-cell carcinoma of the head and neck progressing on or after platinum-based therapy (LUX-Head \& Neck 1): an open-label, randomised phase 3 trial. Lancet Oncol. 2015;16(5):583-594.

27. Xue C, Huang Y, Huang PY, et al. Phase II study of sorafenib in combination with cisplatin and 5-fluorouracil to treat recurrent or metastatic nasopharyngeal carcinoma. Ann Oncol. 2013;24(4):1055-1061.

28. Chou TC. Drug combination studies and their synergy quantification using the Chou-Talalay method. Cancer Res. 2010;70(2):440-446.

29. Hui EP, Lui VW, Wong CS, et al. Preclinical evaluation of sunitinib as single agent or in combination with chemotherapy in nasopharyngeal carcinoma. Invest New Drugs. 2011;29(6):1123-1131.

30. Li T, Ling YH, Goldman ID, Perez-Soler R. Schedule-dependent cytotoxic synergism of pemetrexed and erlotinib in human non-small cell lung cancer cells. Clin Cancer Res. 2007;13(11):3413-3422.

31. Wu YL, Lee JS, Thongprasert S, et al. Intercalated combination of chemotherapy and erlotinib for patients with advanced stage non-small-cell lung cancer (FASTACT-2): a randomised, double-blind trial. Lancet Oncol. 2013;14(8):777-786.

32. Santos CD, Tijeras-Raballand A, Serova M, et al. Effects of preset sequential administrations of sunitinib and everolimus on tumour differentiation in Caki-1 renal cell carcinoma. Br J Cancer. 2015; 112(1):86-94. 


\section{Supplementary material}

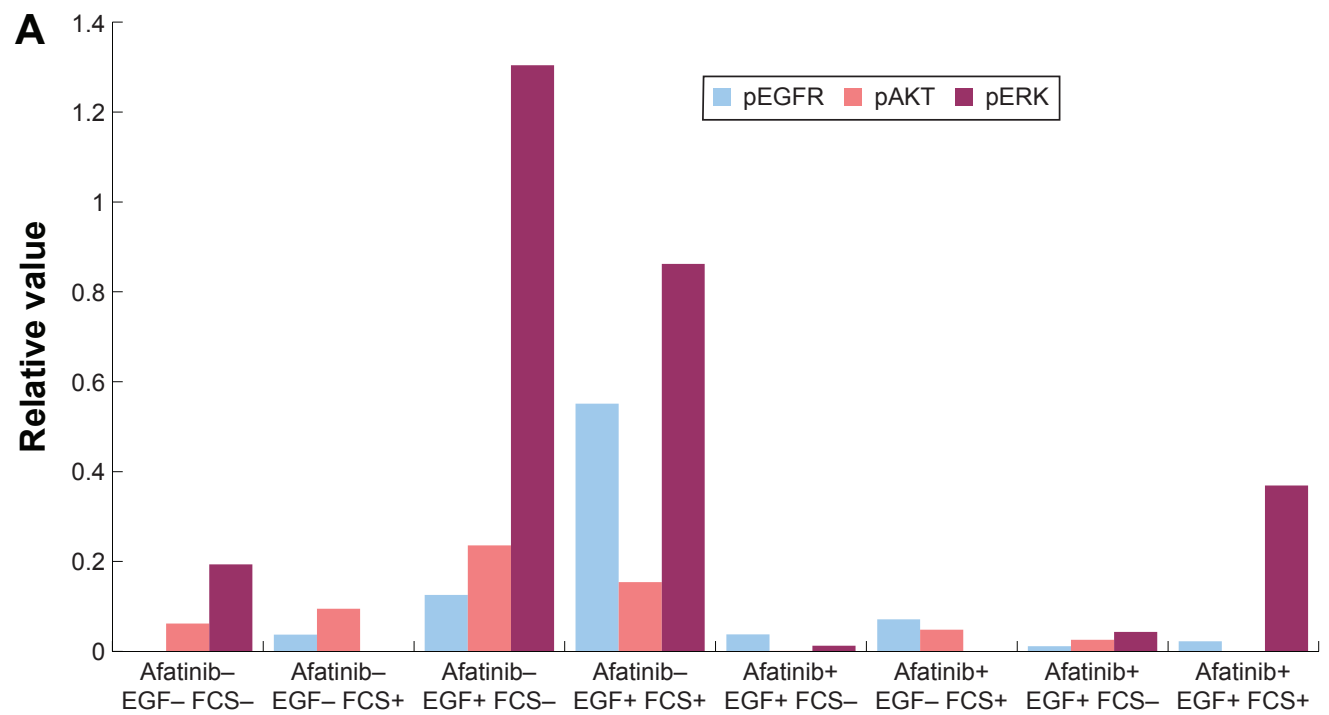

\begin{tabular}{|c|c|c|c|c|c|c|c|}
\hline \multirow[t]{2}{*}{ Groups } & \multicolumn{4}{|c|}{ Gray values } & \multicolumn{3}{|c|}{ Relative values } \\
\hline & GAPDH & pEGFR & PAKT & pERK & pEGFR & PAKT & PERK \\
\hline Afatinib- EGF- FCS- & 105,057 & 0 & 6,527 & 20,339 & 0.00 & 0.06 & 0.19 \\
\hline Afatinib- EGF- FCS+ & 140,012 & 5,225 & 13,272 & 0 & 0.04 & 0.09 & 0.00 \\
\hline Afatinib- EGF+ FCS- & 127,065 & 15,938 & 29,951 & $|65,74|$ & 0.13 & 0.24 & 1.30 \\
\hline Afatinib- EGF+ FCS+ & 153,183 & 84,423 & 23,606 & 132,060 & 0.55 & 0.15 & 0.86 \\
\hline Afatinib+ EGF+ FCS- & 211,044 & 8,015 & 217 & 2,653 & 0.04 & 0.00 & 0.01 \\
\hline Afatinib+ EGF- FCS+ & 150,069 & 10,702 & 7,277 & 0 & 0.07 & 0.05 & 0.00 \\
\hline Afatinib+ EGF+ FCS- & 106,927 & 1,239 & 2,788 & 4,655 & 0.01 & 0.03 & 0.04 \\
\hline Afatinib+ EGF+ FCS+ & 94,106 & 2,134 & 0 & 34,724 & 0.02 & 0.00 & 0.37 \\
\hline
\end{tabular}

Figure SI The relative quantity of phosphorylated EGFR, AKT, and ERK in HNE-I cell line.

Notes: (A) Statistical graph of the relative quantity. (B) The gray values and the relative values.

Abbreviations: EGF, epidermal growth factor; ERK, extracellular regulated protein kinase; FCS, fetal calf serum; GADPH, glyceraldehyde-3-phosphate dehydrogenase; HNE-I, human NPC cell line; pEGFR, phosphorylated epidermal growth factor receptor; $p$, phosphorylated.

\section{Publish your work in this journal}

Drug Design, Development and Therapy is an international, peerreviewed open-access journal that spans the spectrum of drug design and development through to clinical applications. Clinical outcomes, patient safety, and programs for the development and effective, safe, and sustained use of medicines are a feature of the journal, which has also been accepted for indexing on PubMed Central. The manuscript management system is completely online and includes a very quick and fair peer-review system, which is all easy to use. Visit http://www.dovepress.com/testimonials.php to read real quotes from published authors. 